\title{
Geoscience Laser Altimetry System (GLAS) On-Orbit Flight Report on the Propylene Loop Heat Pipes (LHPs)
}

\author{
Charles L. Baker (GSFC), Eric W. Grob (GSFC), Thomas V. McCarthy (GSFC), \\ Michael N. Nikitkin (Swales Aerospace), Walter C. Ancarrow (WCA Inc.)
}

\begin{abstract}
The Geoscience Laser Altimetry System (GLAS) instrument which is the sole instrument on ICESat was launched on January 12, 2003. GLAS utilizes two actively controlled propylene Loop Heat Pipes (LHPs) as the core of its thermal system. The LHPs started quickly when the Dale Ohm starter heaters were powered and have as designed. The low control heater power and on-orbit tight temperature control appear independent of gravity effects when comparing ground testing to flight data. The use of coupling blocks was also unique to these LHPs. Their application reduced control heater power by reducing the subcooling from the radiator. The effectiveness in reducing subcooling of the coupler blocks decreased during flight from ground testing, but internal thermal isolation in the compensation chamber between the subcooled returning liquid increased in flight resulting in no net increase in control heater power versus ground measurements. Overall the application of LHPs in the thermal system for GLAS met instrument requirements and provided flexibility for the overall system as last minute requirements became known.
\end{abstract}


Geoscience Laser Altimetry System (GLAS) On-Orbit Flight

Report on the Propylene Loop Heat Pipes (LHPs)

International Two-Phase Workshop

September 15-17, 2003

Charles Baker, GSFC

Eric Grob, GSFC

Tom McCarthy, GSFC

Michael Nikitkin, Swales

Walter Ancarrow, WCA Inc. 


\section{ICESat Mission}

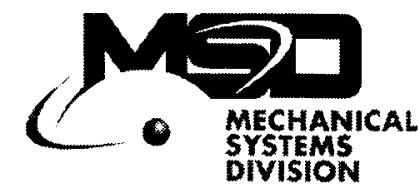

- GLAS is the sole instrument on ICESat, Laser system to measure ice thickness which launched on January 12, 2003

- Orbit - LEO, $600 \mathrm{~km}, 94^{\circ}$ inclination

- Not sun-synchronous; $\sim 0.5^{\circ}$ per day precession rate

- SC yaw maneuvers at specific beta angles to maintain array illumination

- science prohibits continuous yaw steering during data collection

- numerous thermal attitudes consider

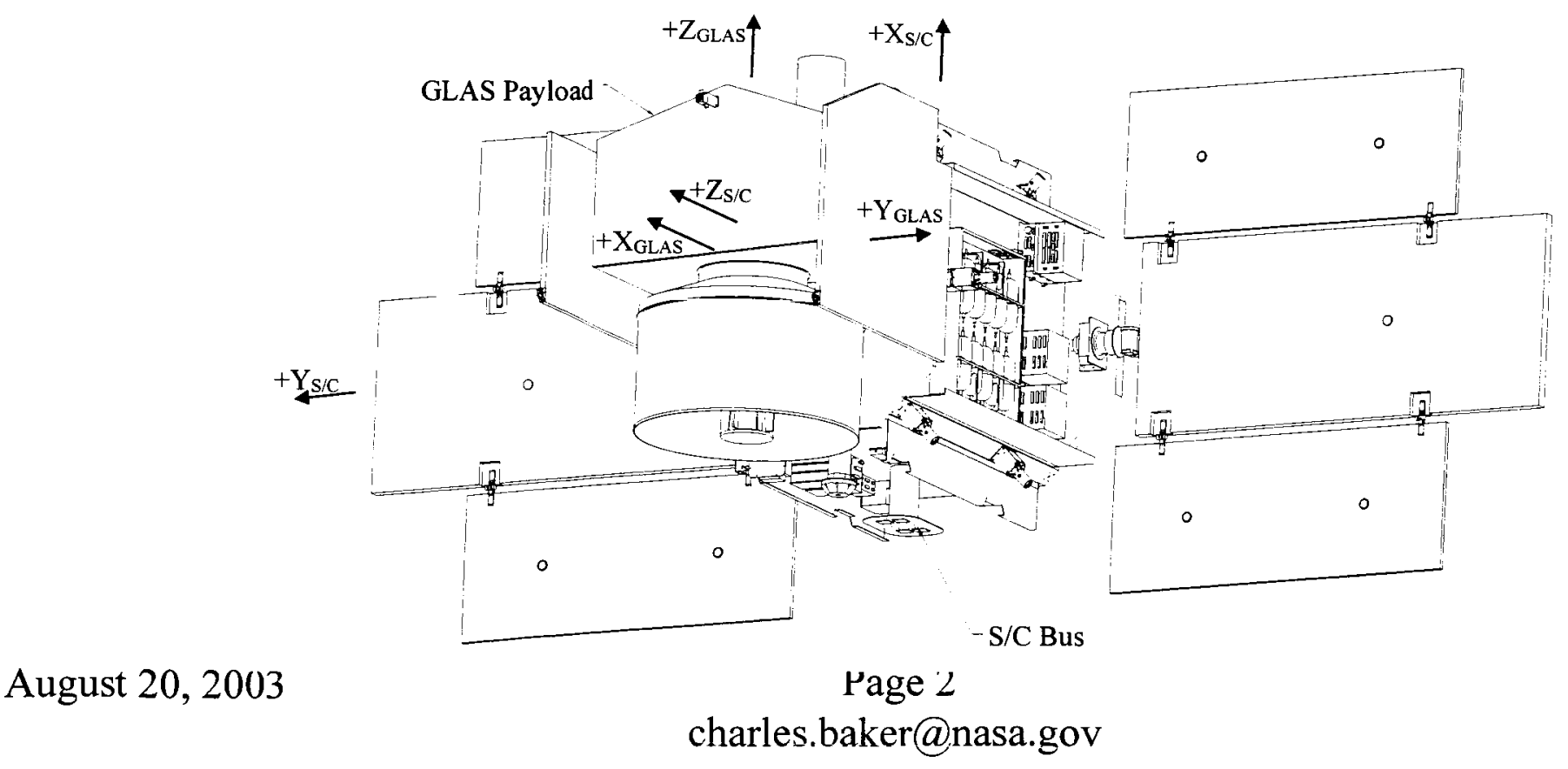




\section{LHPs on GLAS}

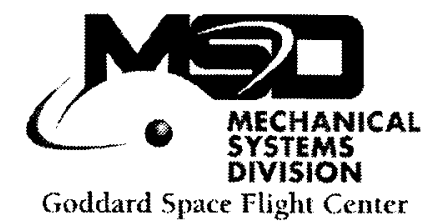

- These two GLAS LHPs are NASA's first LHP application on a flight mission

- The LHPs are running well and exceeding all of their performance specifications

- These LHPs are the first known US non-military use of Propylene as a working fluid in a flight LHP

- These LHPs are the first known tight temperature control LHPs in-flight

- These LHPs utilize passive coupler blocks to minimize subcooling in the cold case which lowers the required control heater power

- The LHPs on GLAS enabled us to do:

- Adjust Instrument temperature late in the schedule to resolve a mis-alignment issue - otherwise a risky boresite alignment mechanism would have needed to be added post environment testing

- Adjustment of the operating temperature within the instrument allows fine-tune alignment of the Instrument optical system

- Maintain tight control during operation for low control heater power and de-couple the radiators during survival cases 


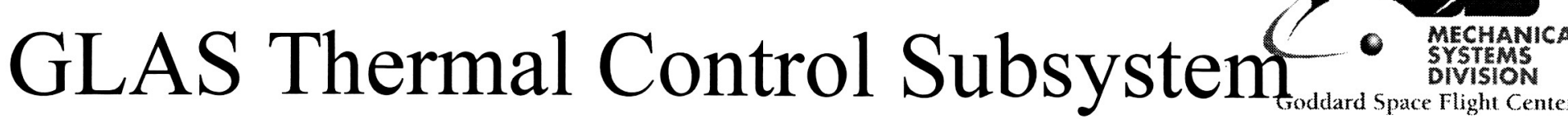

Loop Heat Pipes (propylene): 2

CCHP (ammonia): 10

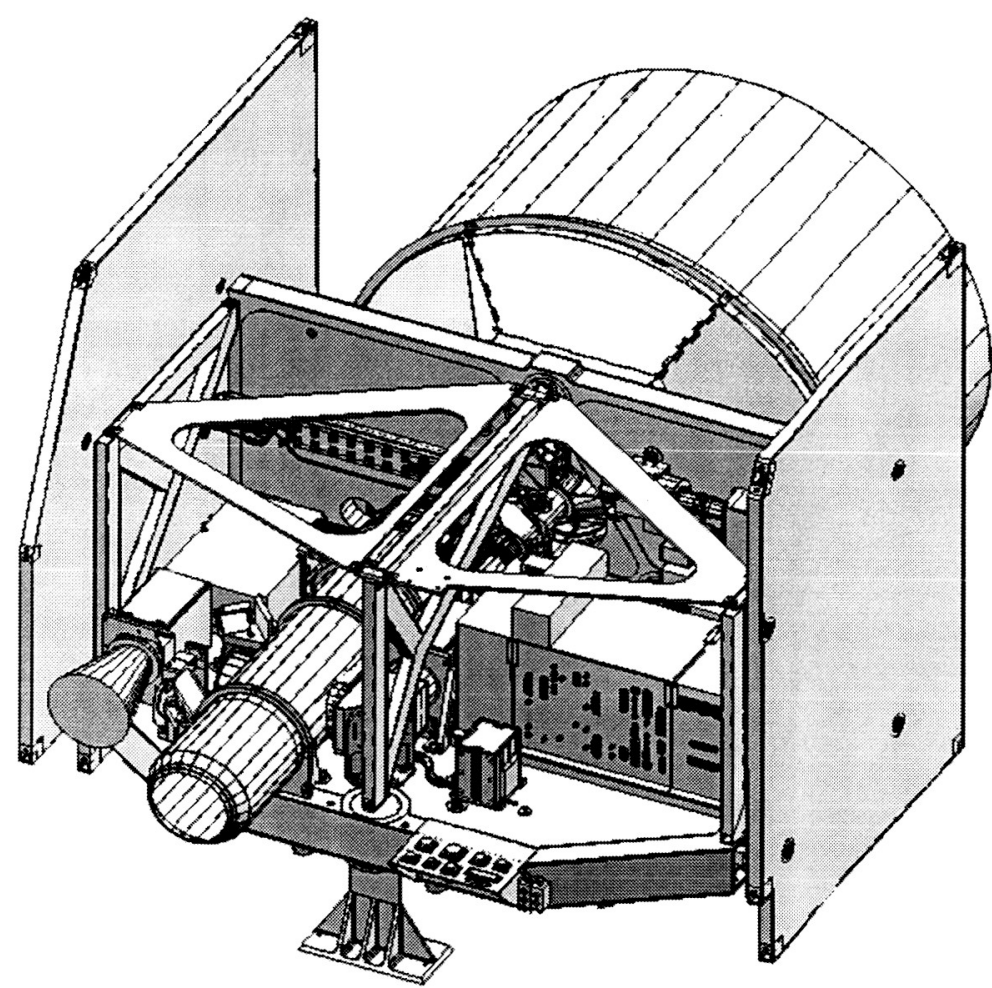

August 20, 2003

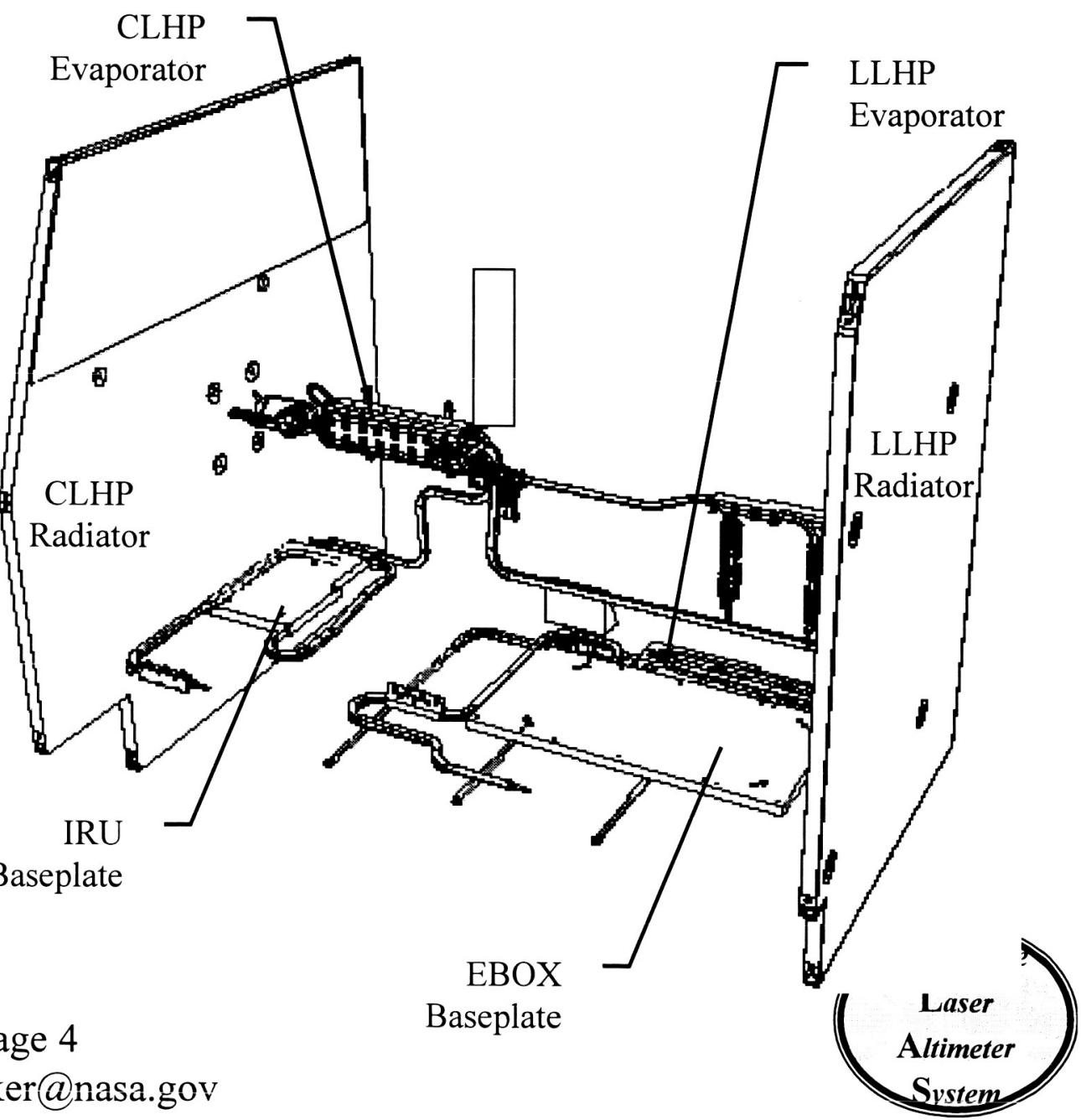

charles.baker@nasa.gov

Sustem 


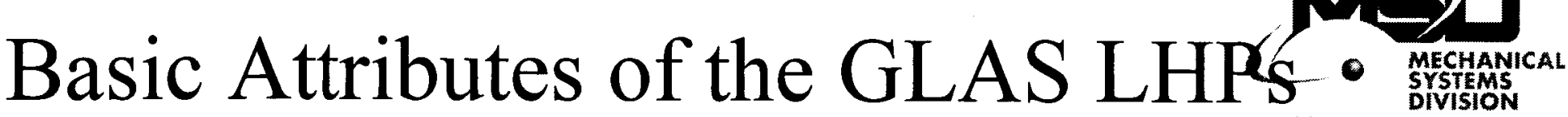

-Propylene is the working fluid so that survival heaters are not required on the radiator (propylene freezes below $-120^{\circ} \mathrm{C}$ )

-LHP System Details:

-Startup Heaters (Dale Ohm) for startup

-58W for $60 \mathrm{Kg}$ of thermal mass CLHP

-29 W for $45 \mathrm{Kg}$ of thermal mass LLHP

- Operational on/off control heaters on compensation chamber are $22 \mathrm{~W}$ (used for temperature control)

-Survival thermostatic heaters for LHP shut down on compensation chamber are $22 \mathrm{~W}$

-LHP Evaporators interfaced directly to a heat pipe network coupling the powered

components to the LHP

-8 - 1" Coupler blocks between the vapor line and liquid line were utilized to reduce the liquid line subcooling and hence reduce required control heater power

-LHPs utilized embedded condenser in the honeycomb radiators 


\section{Thermal Instrumentation (CLHP)}
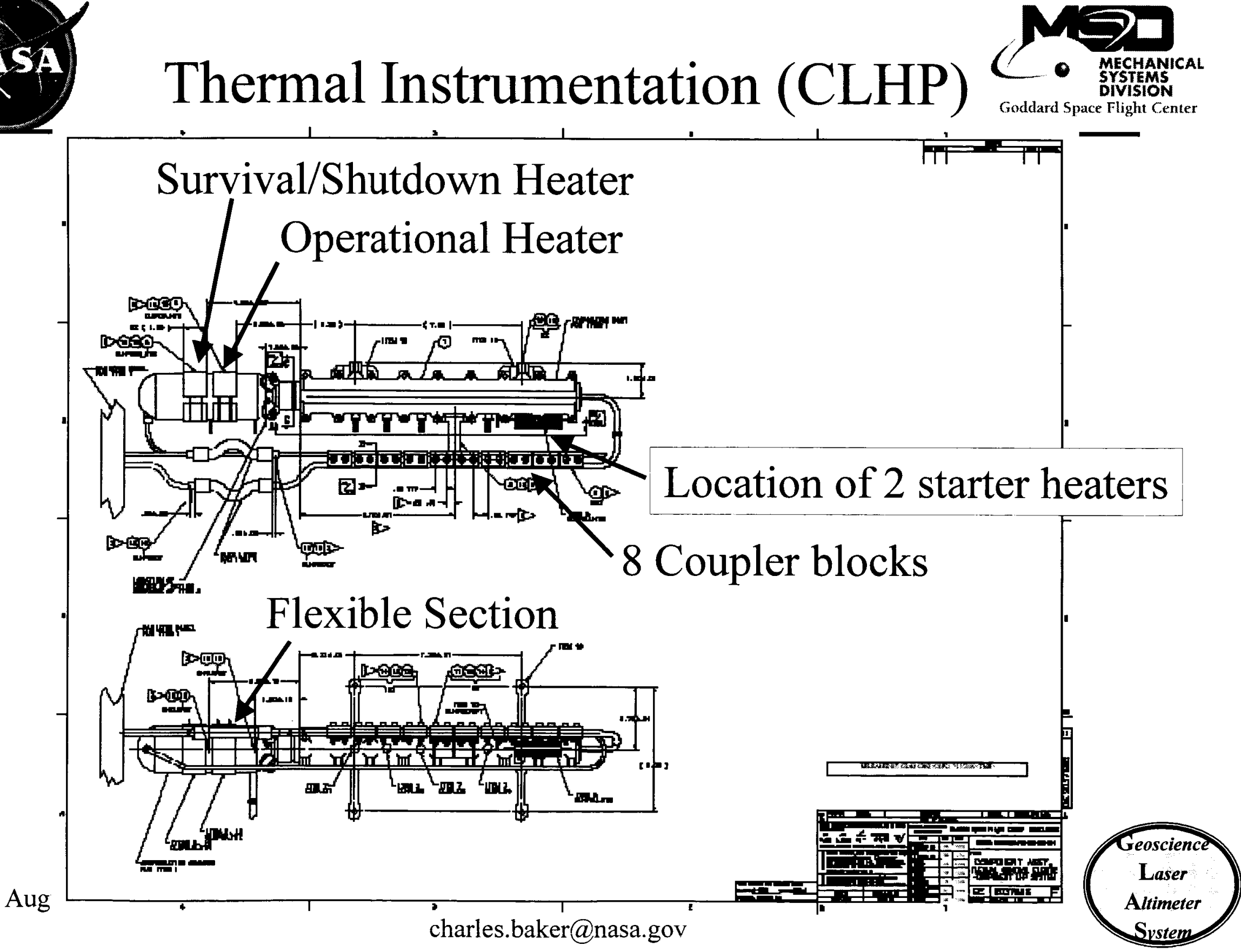

Goddard Space Flight Center 


\section{Thermal Instrumentation (LLHP)}

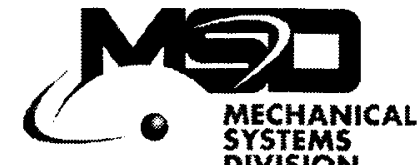

Goddard Space Flight Cen
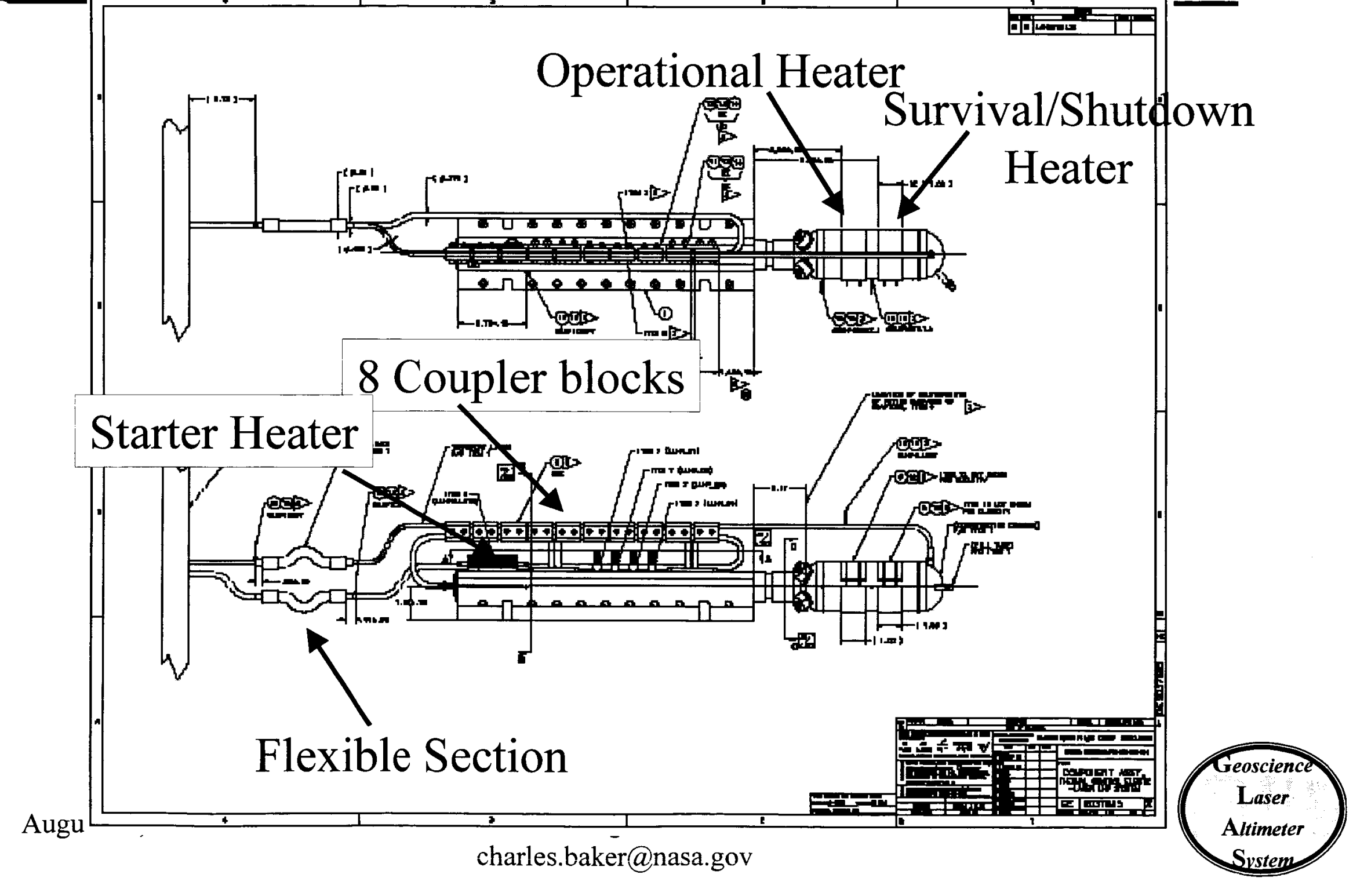


\section{Startup Performance}

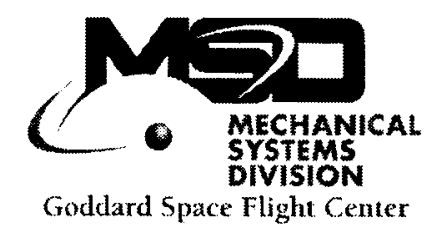

-LHPs did not self-start after launch

-Actual LHP Preconditioning in Flight was "best case" for LHP startup

-LHPs experienced 18 days of settling time prior to being turned on

-CLHP had some cold biasing of its compensation chamber due to a view of a cold radiator $\left(\sim-30^{\circ} \mathrm{C}\right)$ but the CLHPs survival thermostats never were cold enough to click on prior to the LHP starting

-LLHP's survival heater had cycled on and off at the beginning of the mission, but when the LLHP was started, the environment was warm enough so that no heaters had cycled for days prior to the LHP starting

-Both LHPs started within minutes of turning on the starter heater after a temperature spike with the evaporator

-The LLHP started without the starter heater later on in the mission but with $45 \mathrm{~W}$ of heat 


\section{CLHP Startup with $58 \mathrm{~W}$ Str Htr}

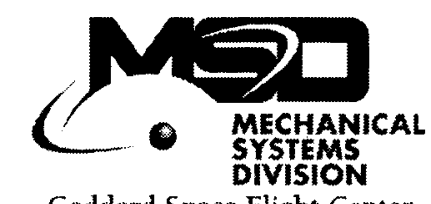

Goddard Space Flight Center

GLAS CLHP Transient Data 02/01/03 (CLHP Turn on)

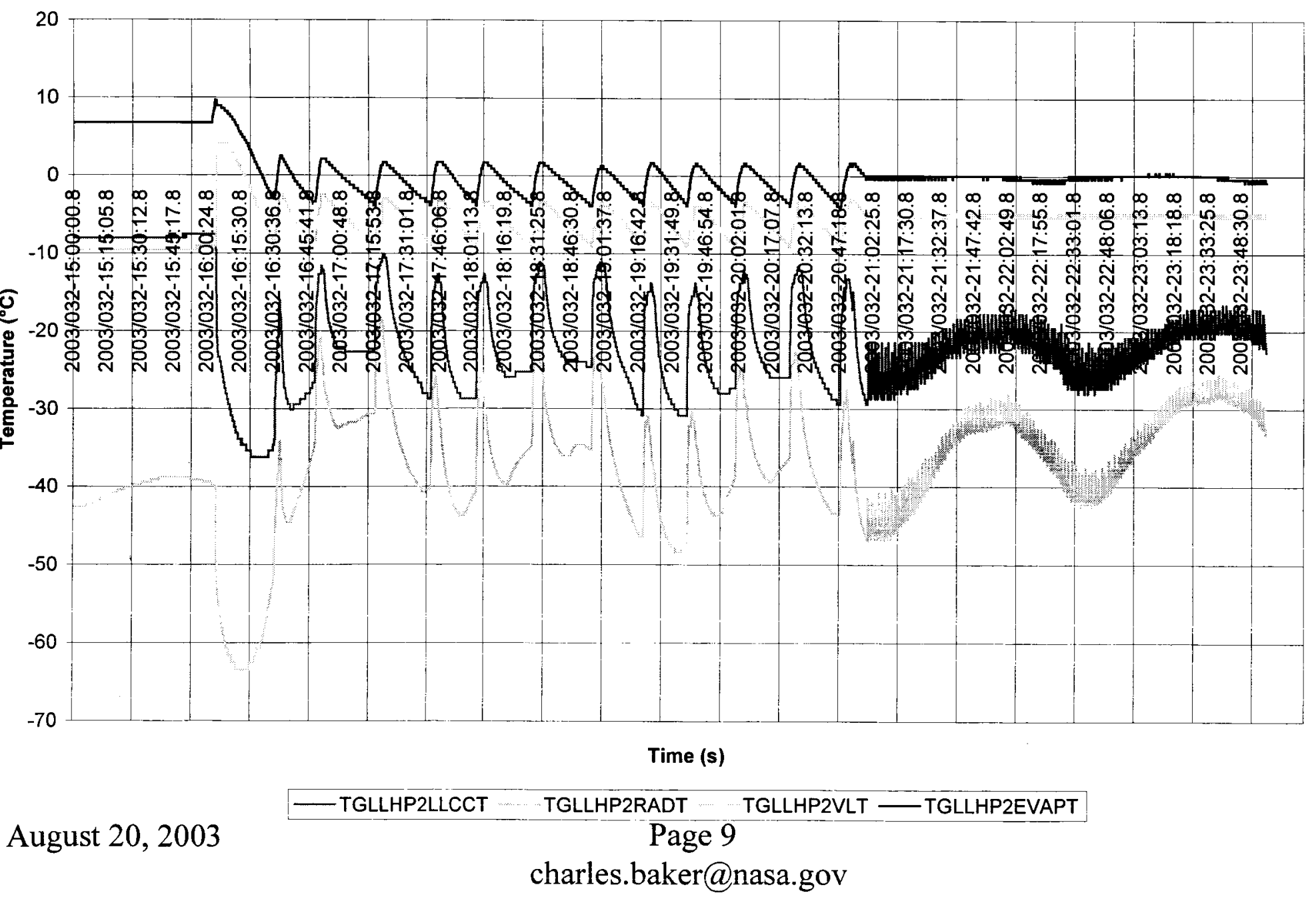




\section{LLHP Startup with 28 W Str Htr}

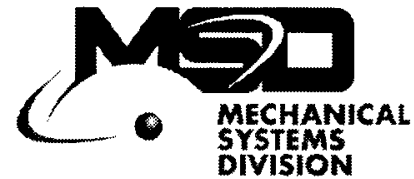

GLAS LLHP Transient Data 02/08/03 (Laser LHP Startup)

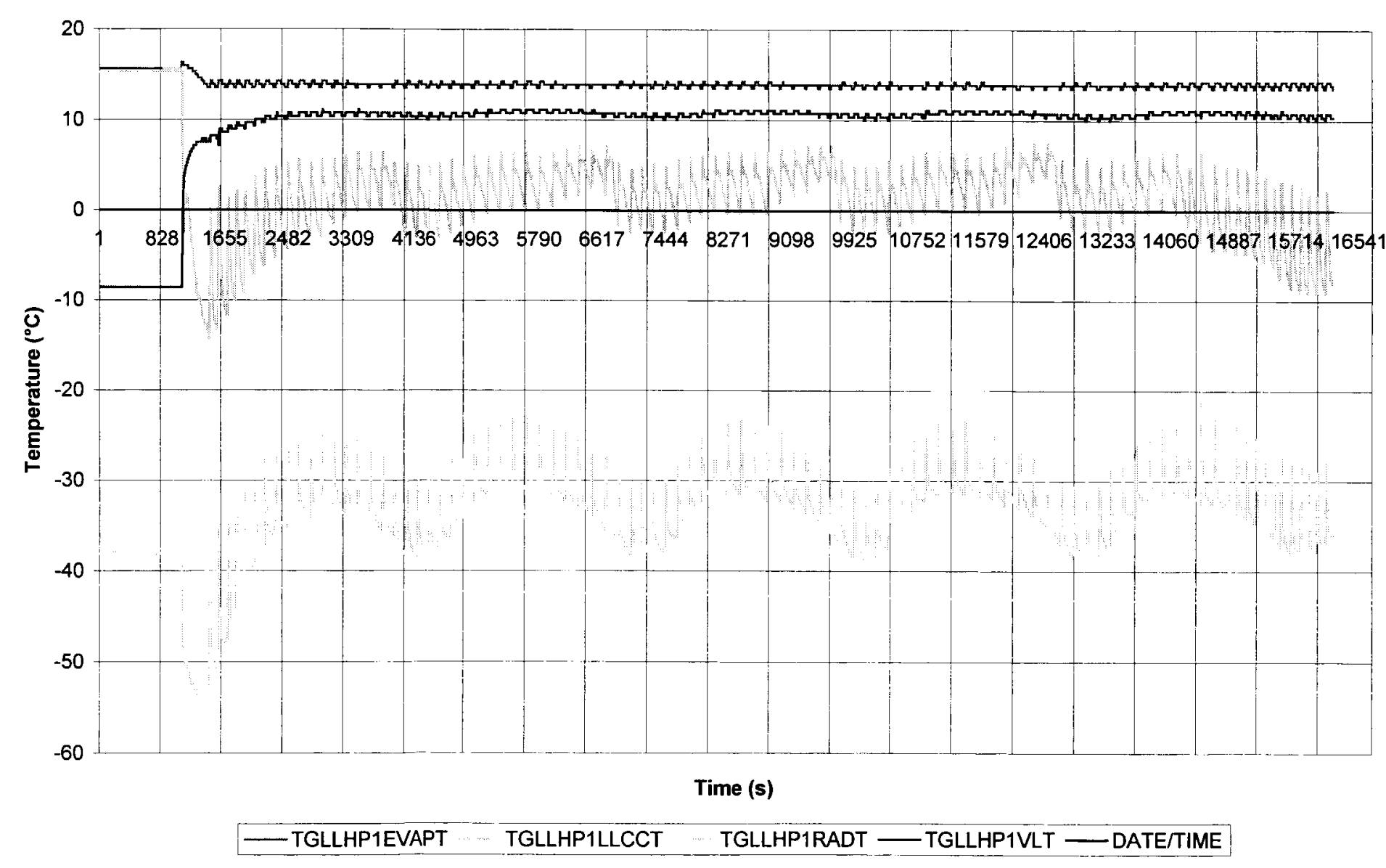

August 20, 2003

Page 10

charles.baker@nasa.gov 


\section{(4) \\ LLHP Startup @ 50 W on Component after slow circulation mode

GLAS LLHP Transient Data 03/29/03 (Laser Turn-on, Turn off warmup heaters)

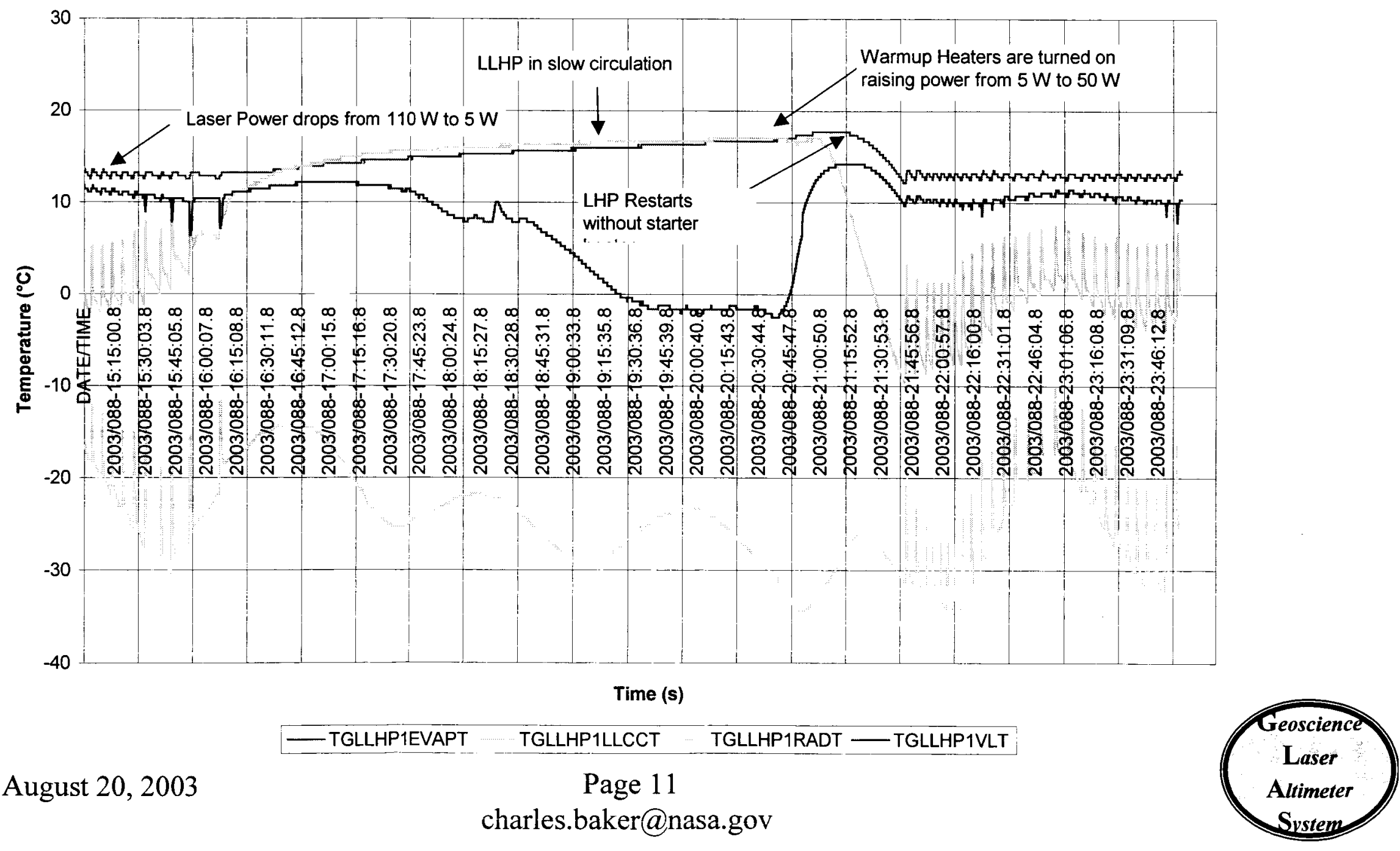




\section{Increasing Setpoints}

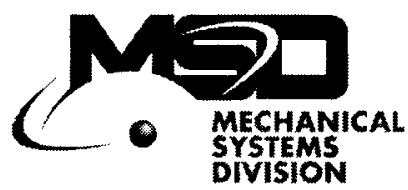

GLAS CLHP Transient Data 04/03/03 (Laser Turn-off, Turn on warmup heaters)

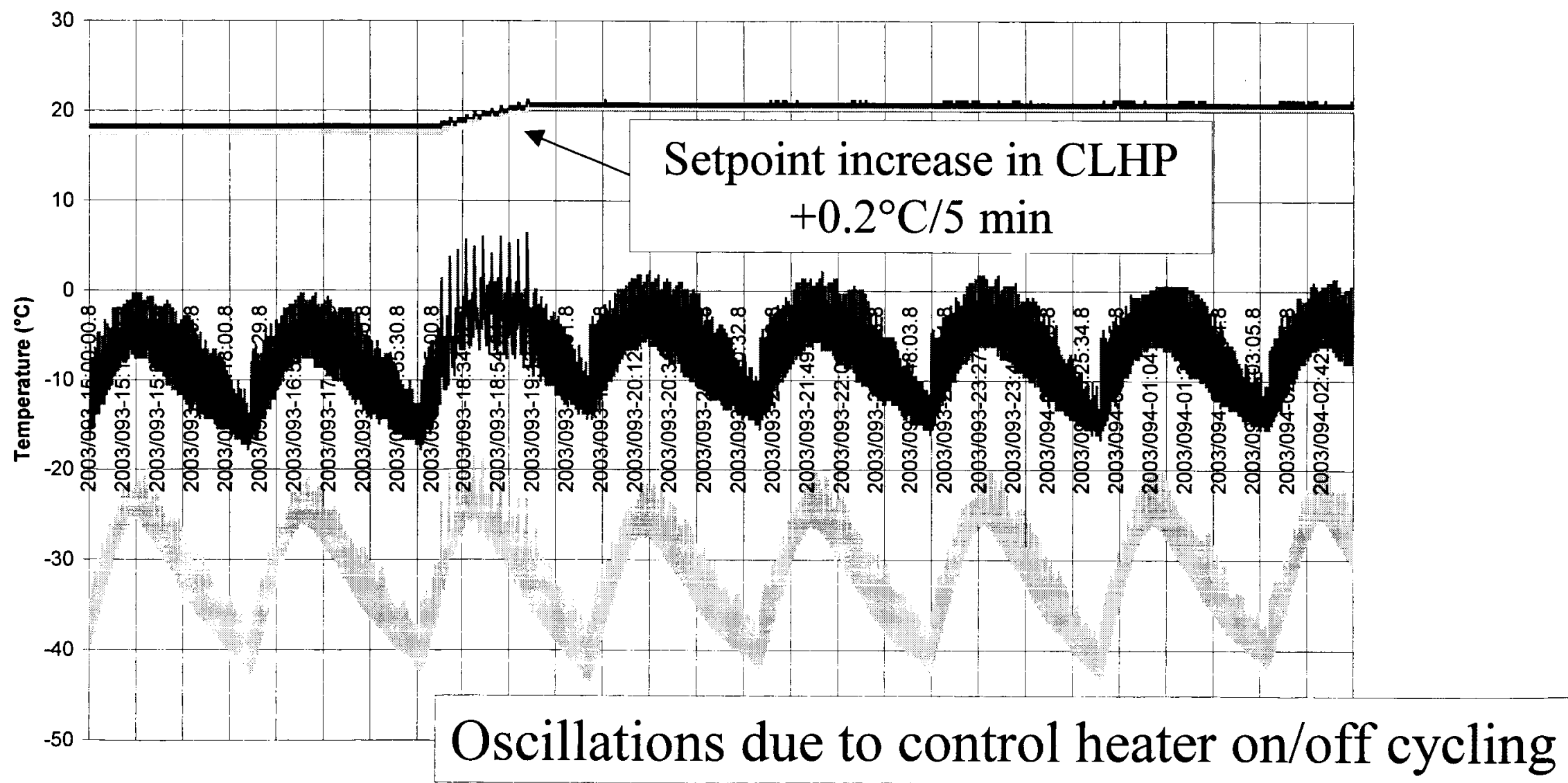

-TGLLHP2LLCCT TGLLHP2RADT TGLLHP2VLT - TGLLHP2EVAPT

August 20, 2003

Page 12

charles.baker@nasa.gov

reoscience

Laser

Altimeter

Sustem 


\section{CLHP Control Performance for 143 W System}

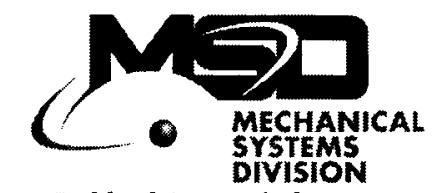

Goddard Space Flight Center

GLAS CLHP Transient Data 02/20/03 (Laser Tum-on, Tum off warmup heaters, all components powered)

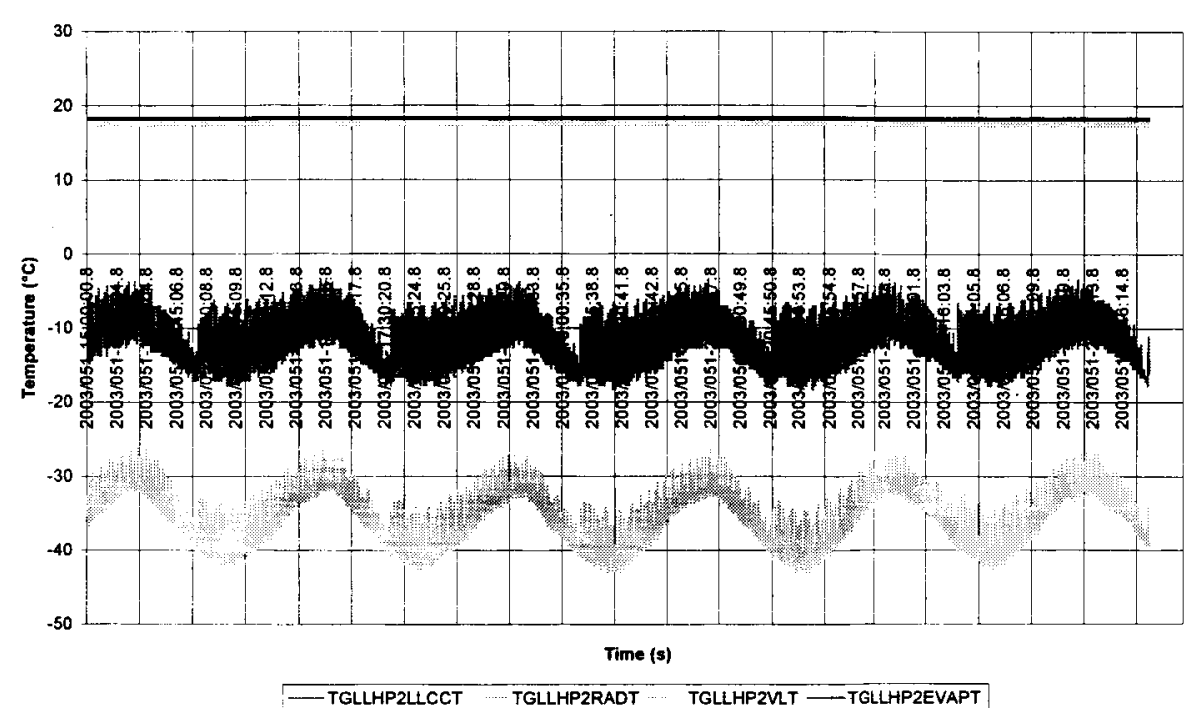

-TGLLHP2LLCCT TGLLHP2RADT TGLLHP2VLT-TGLLHP2EVAPT
GLAS SC Thermistor Transient 02/20/03 (Laser Turn-on, Turn off warmup heaters)
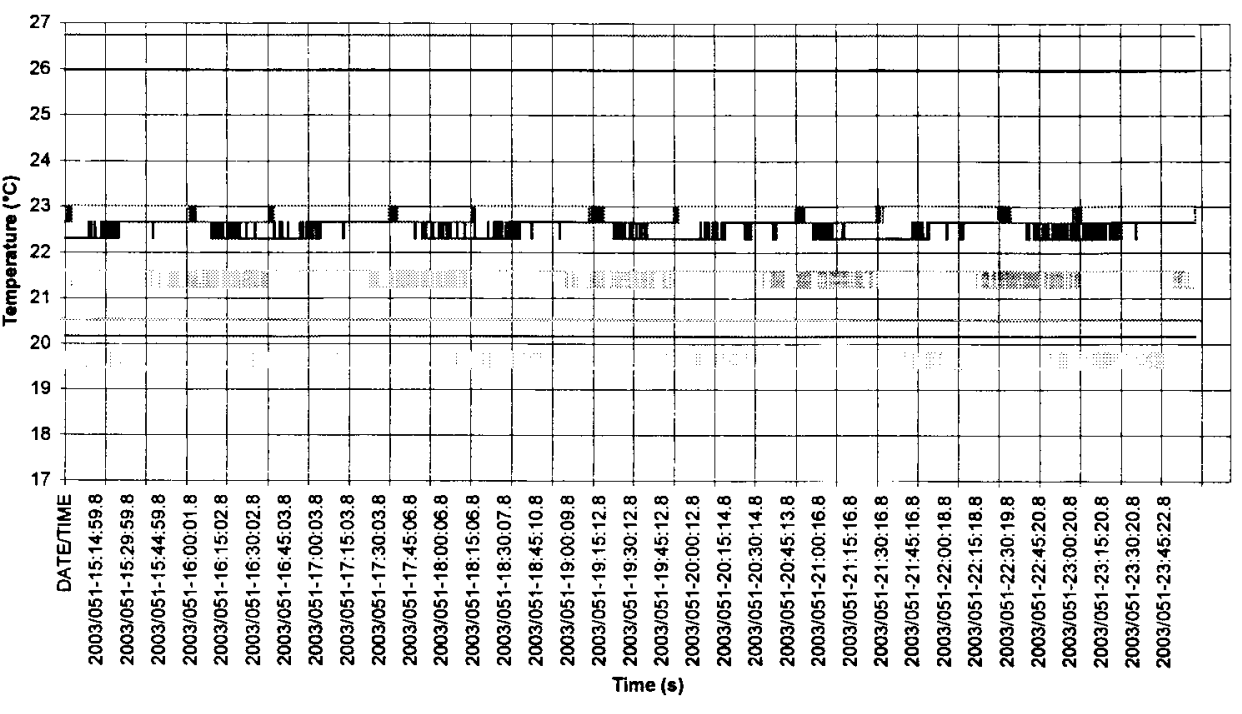

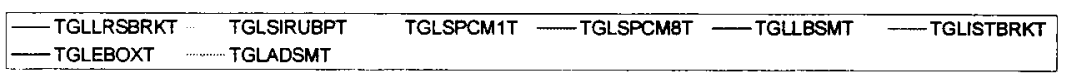

- CLHP Active Control dampens out radiator transients resulting in a $+/-5^{\circ}$ Liquid Return line oscillations to within the less the discretization of the telemetry measurements $+/-0.2$ per orbit on unblanketed components to unmeasurable on blanketed components 
GLAS LLHP Transient Data 02/20/03 (Laser Turn-on. Turn off warmup heaters)

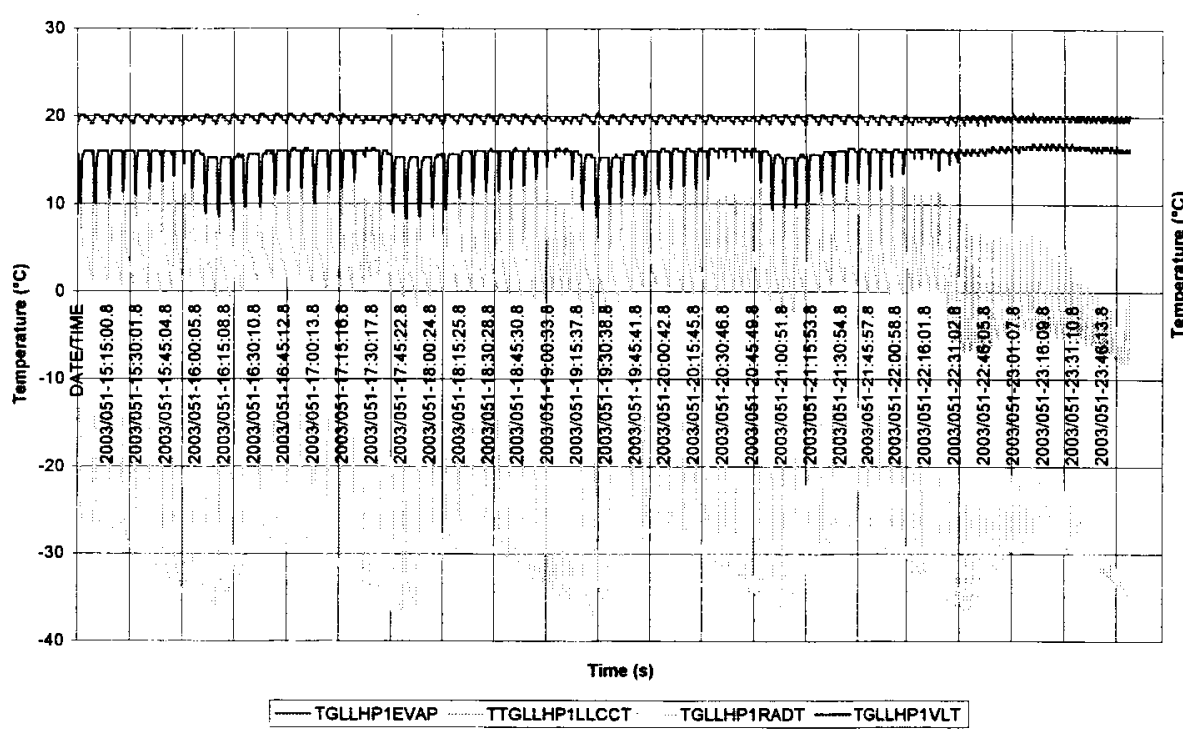

GLAS Laser Transient Data 02/20/03 (Laser Turn-on, Turn off warmup heaters)

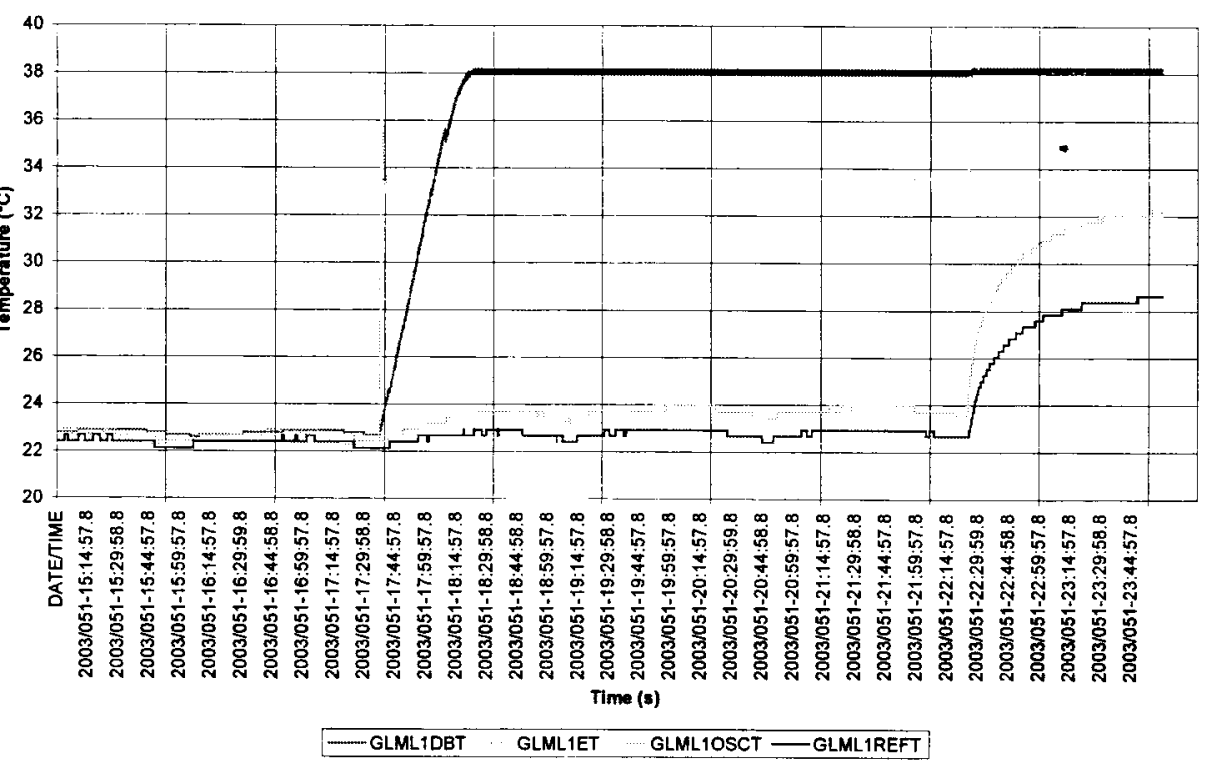

-LLHP Active Control is actually fairly coarse $+/-0.5^{\circ} \mathrm{C}$ per orbit when the LHP has only $45 \mathrm{~W}$ of power input even though the controller has a $0.1^{\circ} \mathrm{C}$ bandwidth between on and off

-The additional oscillation is due to the time constant between the sensor and the heater

August 20, 2003 


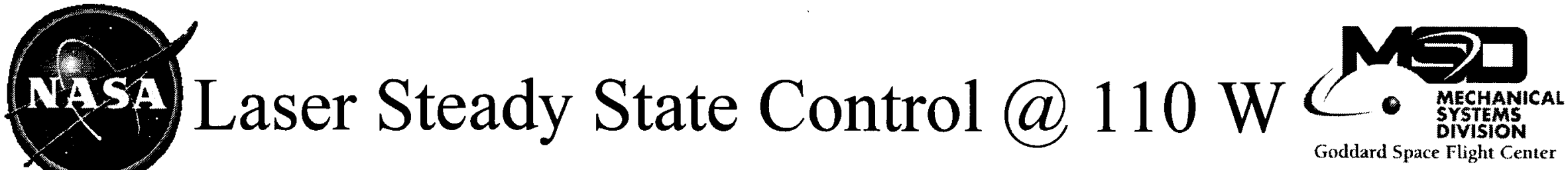

GLAS Laser Transient Data 02/28/03 (Instrument fully powered)

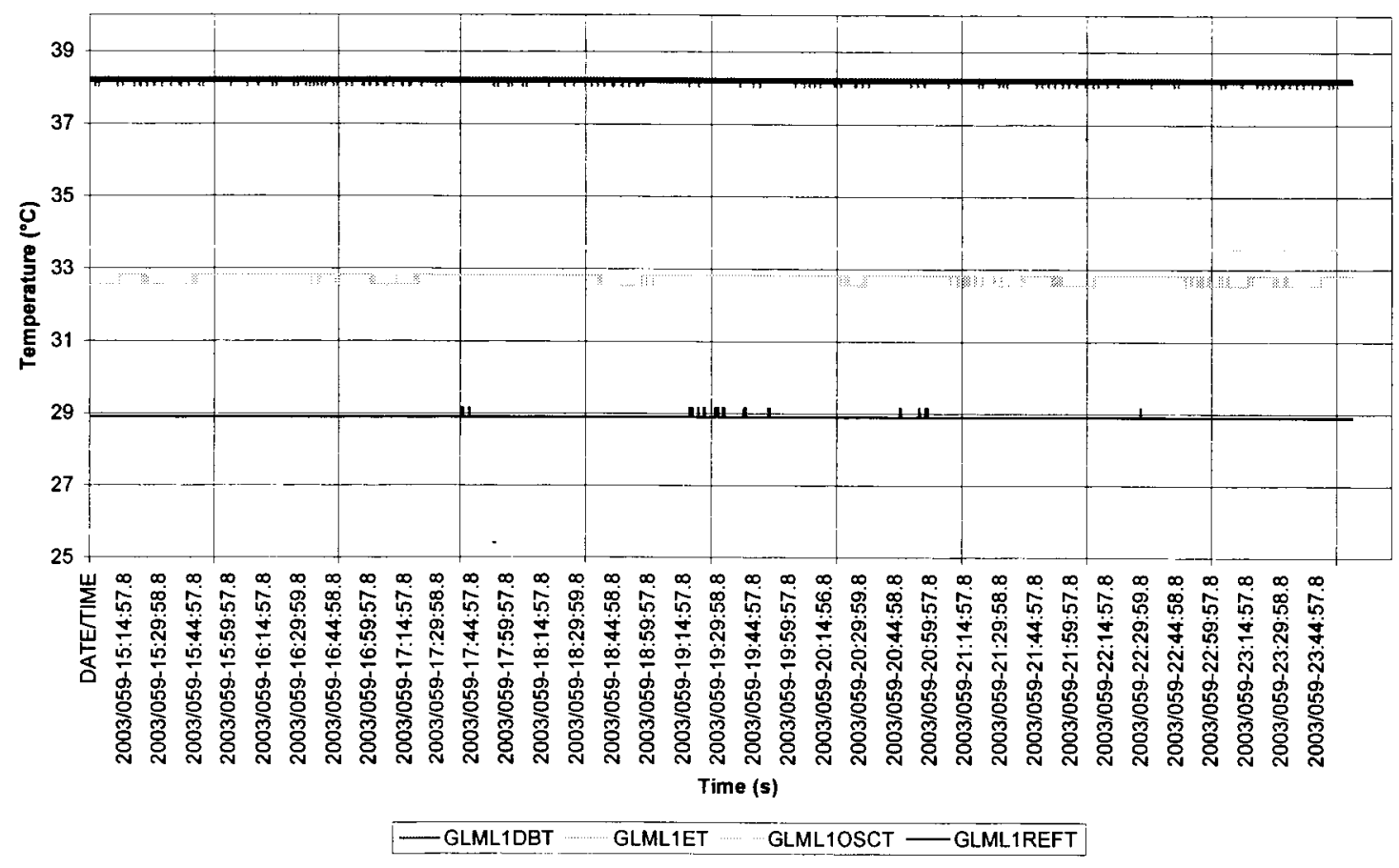

-LLHP Active Control is finer than can be measured in the laser telemetry when the LHP is at full $110 \mathrm{~W}$ of power 


\section{Control Heater Power Measurement}

(ass

Final LLHP Pre-Flight Cases

\begin{tabular}{|c|c|c|c|c|c|c|c|c|c|c|c|}
\hline & Single Mass Power (M) & CC Setpoint $\left({ }^{\circ} \mathrm{C}\right)$ & Thermal Case & Power Measured (M) & $\begin{array}{l}\text { Q model calc from } \\
\text { return line }\end{array}$ & Total Subcooling & TC31 & TC32 & TC32-TC31 & TCCC-TC32 & $\begin{array}{c}\text { Cper block (W/K) } \\
\text { where } 0.034 \text { is laminar } \\
\text { flow }\end{array}$ \\
\hline 1 & 120 & 16.3 & Hot/SS & & 4.2 & & -23 & -1 & 22 & 17.3 & 0.068 \\
\hline 2 & 100 & 16.9 & Cold/SS & & 5.3 & & -35 & -5 & 30 & 21.9 & 0.056 \\
\hline 3 & 100 & 32.6 & Cold/SS & & 6.7 & & -41 & 5 & 46 & 27.6 & 0.06 \\
\hline 4 & 120.3 & 16.2 & Hot/SS & & 3.9 & & -22 & 0 & 22 & 16.2 & 0.072 \\
\hline 5 & 100 & 27 & Cold/SS & & 6.3 & & -43 & 1 & 44 & 26 & 0.061 \\
\hline
\end{tabular}

Final CLHP Pre-Flight Cases

\begin{tabular}{c|c}
6 & 200 \\
7 & 129 \\
8 & 129 \\
9 & 200 \\
10 & 129
\end{tabular}

\begin{tabular}{|c|c|}
\hline 10.4 & Hot/SS \\
10 & Cold/SS \\
15.8 & Cold/SS \\
9.1 & Hot/SS \\
10.5 & Cold/SS \\
\hline
\end{tabular}
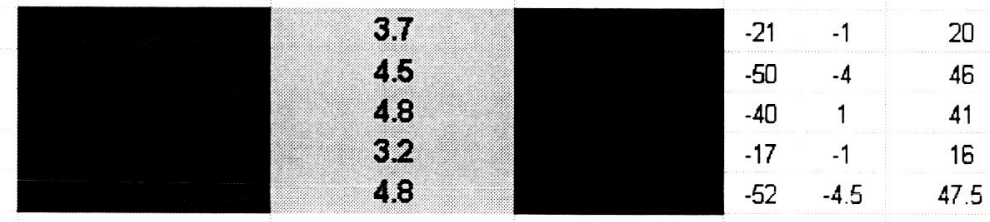

$$
\begin{gathered}
11.4 \\
14 \\
14.8 \\
10.1 \\
15
\end{gathered}
$$

0.146

0.123

0.115

0.139

0.119

Final LLHP Flight Cases

$\begin{array}{ccc}1 & 2003 / 051 & 45 \\ 2 & 2003 / 059 & 110 \\ 3 & 2003 / 079 & 110 \\ 4 & 2003 / 082 & 110 \\ 5 & 2003 / 091 & 45\end{array}$

19.5
19.5
19.5
19.5
12.5
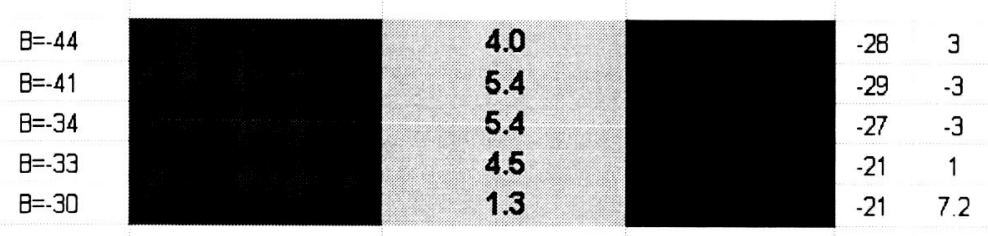

31
26
24
22
28.2

$\begin{array}{ll}16.5 & 0.033 \\ 22.5 & 0.058 \\ 22.5 & 0.055 \\ 18.5 & 0.063 \\ 5.3 & 0.029\end{array}$

Final CLHP Flight Cases

\begin{tabular}{ll|l}
1 & $2003 / 051$ & 143 \\
2 & $2003 / 059$ & 143 \\
3 & $2003 / 079$ & 143 \\
4 & $2003 / 082$ & 143 \\
5 & $2003 / 091$ & 143
\end{tabular}

17.8
17.8
17.8
17.8
17.8

$B=-44$
$B=-41$
$B=-34$
$B=-33$
$B=-30$
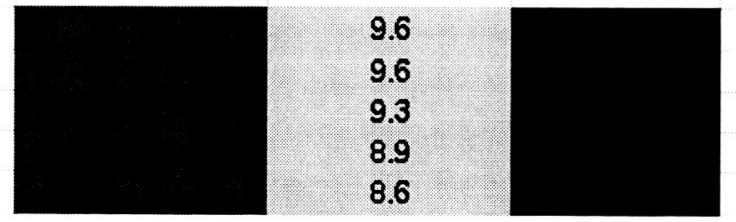

$\begin{array}{lc}-35 & -12 \\ -35 & -12 \\ -35 & -11 \\ -31 & -10 \\ -32 & -9\end{array}$

23
23
24
21
23

29.8
29.8
28.8
27.8
26.

0.051

0.051

0.055

0.052

0.057

August 20, 2003

Page 16

charles.baker@nasa.gov
Geoscience

Laser

Altimeter

S sstem 


\section{Effectiveness of Coupler Blocks on Reducing Control Heater Power}

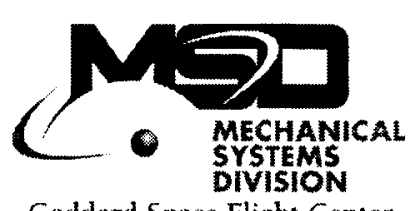

Goddard Space Flight Center

- If the flow thru the liquid line is assumed to be laminar (meaning that the mass flow rate is stable) the calculated conductance thru each block would be 0.034 $\mathrm{W} / \mathrm{K}$

- This number corresponds well to the LLHP when it is operating at $45 \mathrm{~W}$ (note this page's numbers are taken from the chart on the proceeding page)

- The flight effectiveness of the Coupler blocks is lower indicating closer to laminar flow than ground with 1 -g effects

- The coupler blocks essentially reduce the subcooling by about half

$$
\begin{aligned}
& \text { Flight } \\
& (\mathrm{W} / \mathrm{K})
\end{aligned}
$$

\begin{tabular}{|c|c|}
\hline Cperblock & \\
\hline 0.033 & \\
0.058 & mean \\
0.055 & 0.0476 \\
0.063 & std dev \\
0.029 & 0.015485 \\
\hline
\end{tabular}

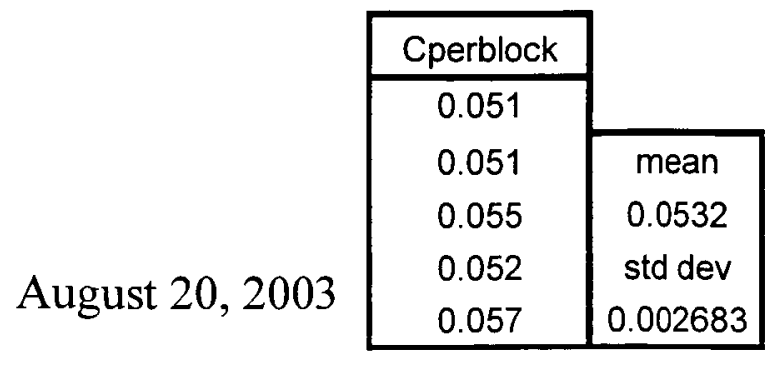
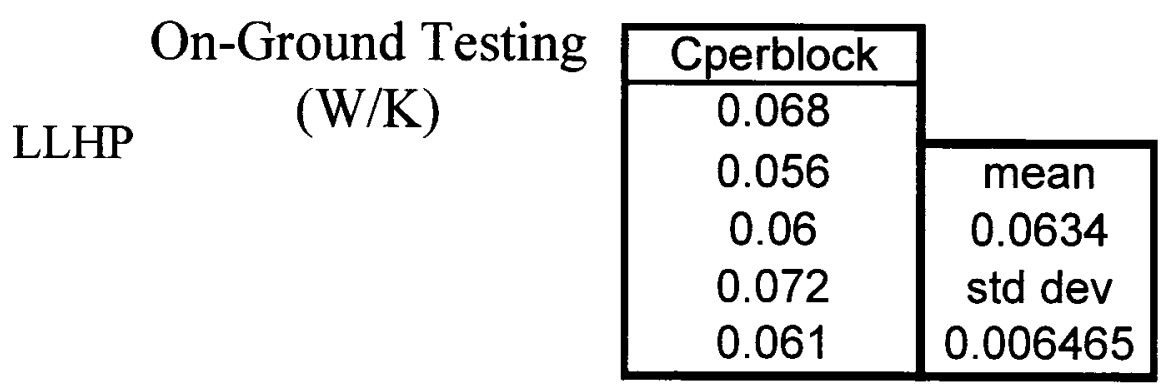

CLHP

Page 17 charles.baker@nasa.gov reoscience Laser Altimeter 


\section{Control Heater Power Required Test to Flight}

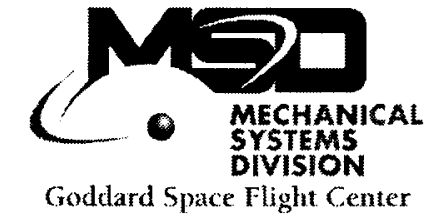

- Despite the blocks being less effective in flight, the overall control heater power was about the same as on the ground when comparing similar cases i.e CLHP power measured comparison of Beta angle 33 before the yaw (Hottest attitude) at $4 \mathrm{~W}$ versus Hot/SS at Hot/SS around $3.7 \mathrm{~W}$

- Because the blocks are less effective and the heater power is the same, it must mean the liquid return line is better insulated from the 2-phase bubbles in the compensation chamber during 0 -g Flight versus 1 -g test which results in:

- less of the subcooling power being transferred to the compensation chamber thru condensing vapor bubbles

- therefore due to conservation of energy, most of the subcooling is entering the wick and being sensibly heated to saturation temperature before evaporating 


\section{Heat Pipe + LHP Conductance Test to Flight}

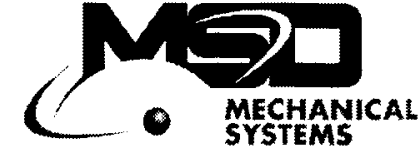

SYSTEMS

Goddard Space Flight Center

\begin{tabular}{|l|c|c|c|}
\hline MDT & dT from CLHP temp & dT from LHP CC temps & Percentage Decrease \\
\hline & Flight & Hot Soak Observatory & $(\%)$ \\
\hline SC thermistors & & & \\
\hline TGLISTBRKT (Star Tracker Brackt) & & & $17 \%$ \\
\hline TGLLRSBRKT (LRS Brackt) & 5.4 & 6.5 & $17 \%$ \\
\hline TGLSPCM1T (Lidar Det SPCM 1) & 9.4 & & \\
\hline TGLSPCM8T (Lidar Det SPCM 8) & & & $86 \%$ \\
\hline TGLLHP1EVAPT (LLHP Evap) & & 2.2 & $50 \%$ \\
\hline TGLLHP2EVAP2 (CLHP Evap) & 0.3 & 0.9 & $31 \%$ \\
\hline TGLSIRUBPT (IRU Base) & 0.4 & 5.5 & $18 \%$ \\
\hline TGLEBOXT (MEU base) & 3.8 & 10.5 & \\
\hline
\end{tabular}

- Heat Pipe conductances improved from ground testing due to the better liquid feeding of the grooves in the absence of gravitational forces by about $17 \%$

- It also appear that the LHP conductances have improved since the dT has decreased between the evaporator and compensation chamber, but insufficient flight instrumentation (particularly on the heat pipe headers) prevents an accurate statement of how much they improved

- Evaporator Conductance: $\sim 24 \mathrm{~W} / \mathrm{K}$ for DM LHP as compared to 35 to 40 $\mathrm{W} / \mathrm{K}$ for the flight units (in 1-g testing) though not expected to substantially change in flight

August 20, 2003 


\section{Hot versus Cold Case Performance}

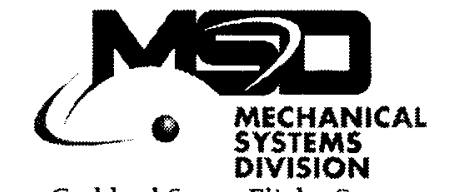

Goddard Space Flight Center

- Component temperatures that are linked via the heat pipe network are independent to the thermal environment of the radiators

- Control heater power does decrease as the condensers warm up 


\section{Conclusion}

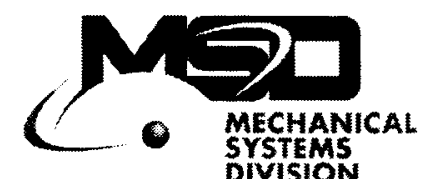

-Use of Propylene LHPs for the GLAS instrument was a good choice for tight control and diode action required for the system

-The LHPs were flexible for late changing requirements

-No major changes between in-flight and on-ground performance 